\title{
Computerized Functional Reach Test to Measure Balance Stability in Elderly Patients With Neurological Disorders
}

\author{
Silvio Scena ${ }^{\mathrm{a}}$, Roberto Steindler ${ }^{\mathrm{a}}$, Moira Ceci ${ }^{\mathrm{b}}$, Stefano Maria Zuccaro ${ }^{\mathrm{b}}$, \\ Eli Carmelic, d
}

\begin{abstract}
Background: The ability to maintain static and dynamic balance is a prerequisite for safe walking and for obtaining functional mobility. For this reason, a reliable and valid means of screening for risk of falls is needed. The functional reach test (FRT) is used in many countries, yet it does not provide some kinematic parameters such as shoulder or pelvic girdles translation. The purpose was to analyze video records measuring of distance, velocity, time length, arm direction and girdles translation while doing FRT.
\end{abstract}

Methods: A cross-sectional, descriptive study was conducted where the above variables were correlated to the mini-mental state examination (MMSE) for mental status and the Tinetti balance assessment test, which have been validated, in order to computerize the FRT (cFRT) for elderly patients with neurological disorders. Eighty patients were tested and 54 were eligible to serve as experimental group. The patients underwent the MMSE, the Tinetti test and the FRT. LAB view software was used to record the FRT performances and to process the videos. The control group consisted of 51 healthy subjects who had been previously tested.

Results: The experimental group was not able to perform the tests as well as the healthy control subjects. The video camera provided valuable kinematic results such as bending down while performing the forward reach test.

Conclusions: Instead of manual measurement, we proposed to use a cheap with fair resolution web camera to accurately estimate the FRT. The kinematic parameters were correlated with Tinetti and MMSE scores. The performance values established in this study indicate that the cFRT is a reliable and valid assessment, which provides more ac-

Manuscript accepted for publication July 18, 2016

aDepartment of Mechanical and Aerospace Engineering, University of Rome "La Sapienza", Via Eudossiana 18, 00184 Roma, Italy

bIsraelitic Hospital of Rome, Via Fulda, 14, 00148 Roma, Italy

'Physical Therapy Department, Faculty of Social Welfare and Health, University of Haifa, 3498838, Israel

${ }^{\mathrm{d} C}$ Corresponding Author: Eli Carmeli, Physical Therapy Department, Faculty of Social Welfare and Health, University of Haifa, 3498838, Israel.

Email: ecarmeli@univ.haifa.ac.il

doi: http://dx.doi.org/10.14740/jocmr2652w curate data than "manual" test about functional reach.

Keywords: Aging; Balance; Brain disorder; Functional reach test; Tinetti balance test

\section{Introduction}

Elderly individuals often suffer from a slow, progressive decrease in cognitive function [1] and from increasing loss of balance related to psycho-sensory-motor slowdown [2]. This cognitive and functional decline has a harmful effect on quality of life and increases the risk of falls and hospitalizations [3]. Falls in particular are a serious public health concern and contribute to considerable morbidity and mortality in older people. Identifying risk factors to minimize the probability of falls or even to prevent falls in the elderly is crucial for both health and social reasons. The use of standardized instruments to measure the health status of patients has been promoted in physical rehabilitation to help develop and implement effective treatment strategies. In the elderly, it is important to assess functional limitations and physical performance, which are used to indicate the impact of disease, impairment, and other risk factors.

Consequently, several clinical tests have been designed to assess balance and mobility, including the timed up and go (TUG) [4] and the Berg balance scale [5]. Some tests, such as gait speed [6], sit-to-stand [7], the functional reach test (FRT) [8], and the Zur balance scale [9] have also been proposed as able to predict falls, particularly among older people. In some tests, particularly gait speed, advantages have been reported in terms of test-retest reliability, sensitivity to change, and applicability to different population groups [10]. Yet, other tests have not been validated in samples of community-dwelling older people, making their predictive accuracy uncertain. Furthermore, the applicability of these studies in clinical practice, both for screening and for monitoring and evaluating change, has not been demonstrated [11].

The FRT is a simple, portable, clinically accepted tool that is used to measure semi-static balance. It is based on analyzing the limits of anterior-posterior stability in the absence of external perturbations, by assessing the maximum displacement (FR, distance in $\mathrm{cm}$ ) a subject can attain without stepping or losing balance. Thus, it integrates biomechanics, postural con- 
trol, and proprioceptive feedback and correlates results of the higher risk of falling. This test has been used to assess semistatic balance in patients with neurological diseases such as Parkinson's, stroke, physical frailty, vestibular dysfunction, and aging. The consequences of the neurological diseases are that most individuals who regain mobility have permanent impairment in dynamic standing balance, which is needed to achieve a normal walking gait. The ability to maintain semistatic and thus, dynamic balance is a prerequisite for safe walking and for obtaining functional mobility. For this reason, a reliable and valid means of screening for risk of falls is needed. Therefore, the aim of this study was to evaluate the dynamic balance ability of elderly patients with neurological impairment using a computerized FRT (cFRT). For that matter, we analyzed video records measuring of distance, velocity, time length, arm direction and girdles translation while doing FRT. The FRT has been shown to assess the problems of patients with Parkinson's disease [12], stroke survival [13] and healthy controls.

Generally, the FRT is measured by an observer with reference to a graduated tape measure $[3,8]$ and does not require any specific equipment. In this study, we used video recordings and software elaborations to computerize the FRT (cFRT) in order to increase diagnostic accuracy by providing additional functional reach parameters.

Moreover, based on these assumptions and on our previous results [14-16], we wanted to measure correlations between the cFRT, the mini-mental state examination (MMSE) [17] and the Tinetti balance test [18].

Our hypothesis was that the video recording and the LAB view software would provide valuable kinematic variables that could be correlated with the Tinetti and MMSE tests.

\section{Materials and Methods}

\section{Subjects}

\section{Sample size}

Based on a $95 \%$ confidence interval, $10 \%$ sampling error, and the patients population size, 60 participants were needed, 40 patients and 20 healthy participants. The sampling frame was taken through convenience and snowball sampling.

A total of 80 patients (38 males and 42 females) with neurological disorders volunteered to participate in this crosssectional descriptive study. They randomly arrived from the outpatient clinic of the Israelitic Hospital of Rome, from February 2014 through September 2015. Inclusion criteria were neurological disorders due to brain trauma, ability to walk independently for 10 min without the need of physical support or support from another person, ability to understand and perform the FRT appropriately (the subject understood the aim of the test, did not lift heels, did not lose balance, and did not need any assistive device (e.g., cane or crutch)). Exclusion criteria were minimal understanding of Italian, less than 65 years old, blind, Parkinson's disease, stroke (CVA), seizures, heart disease, or- thopaedic problems, back pain, muscles contracture, MMSE score less than 22, or sport practice at a competitive level. Only 54 subjects (26 males and 28 females) met the above criteria. The Ethics Committee of the Israelitic Hospital approved the study. Informed consent was obtained from the patients prior to their participation. The results of previous analogous tests on 51 healthy subjects were used as control data. The control groups included 25 healthy subjects (14 males and 11 females) from the Institutes for elderly associated with the Israelitic Hospital of Rome and 26 healthy subjects (10 males and 16 females) from nursing homes in Haifa, Israel. To be eligible to participate in the healthy/control group, subjects had to be independent in activities of daily living, pain free and without any acute inflammation.

\section{Randomization and blindness}

Upon being recruited, each of the patient was given a participator's number, running from 1 to 100 . To avoid fatigue from data collection, all participants with an uneven participator's numbers assigned to a "first group", and participants with even numbers assigned to a "second group". The "first group" started the evaluation process in "order A" (i.e. MMSE, Tinetti test, and FRT, and lastly cFRT), and the "second group" started the evaluation process in opposite way ("order B") beginning with cFRT, FRT, Tinetti and MMSE as the last test. All tests were taken by an experienced physical therapist that served as a research assistant and was not involved in analyzing the data.

\section{Outcome measures}

\section{MMSE}

The MMSE was used to study correlations between cognitive abilities and physical performance. The MMSE is easy to perform; it takes about 5 min and consists of 11 items regarding time-space orientation, short-term memory, ability to calculate, and constructive praxis. The total score is the sum of the results of each item and ranges from 0 (maximum cognitive deficit) to 30 (cognitive deficit absence). The threshold score is 23 - 24 and the most subjects without dementia seldom reach a low score.

\section{Tinetti test}

The Tinetti balance test was chosen because it is a functional test. It is relatively quick to administer (10 - $15 \mathrm{~min})$ and does not require specialized equipment, making it suitable for use in clinical settings. It was used to assess the balance and gait of elderly subjects, cognitively healthy or with mild-to-moderate dementia. It consists of 17 items (score ranging from 0 to 2 for each item). There are two subtests, one for balance (sit-to-stand from sitting) and one for gait (beginning gait, the characteristics of the step, the ability to turn around $360^{\circ}$ ). The total score, from 0 to 28 , is the sum of the scores of the 


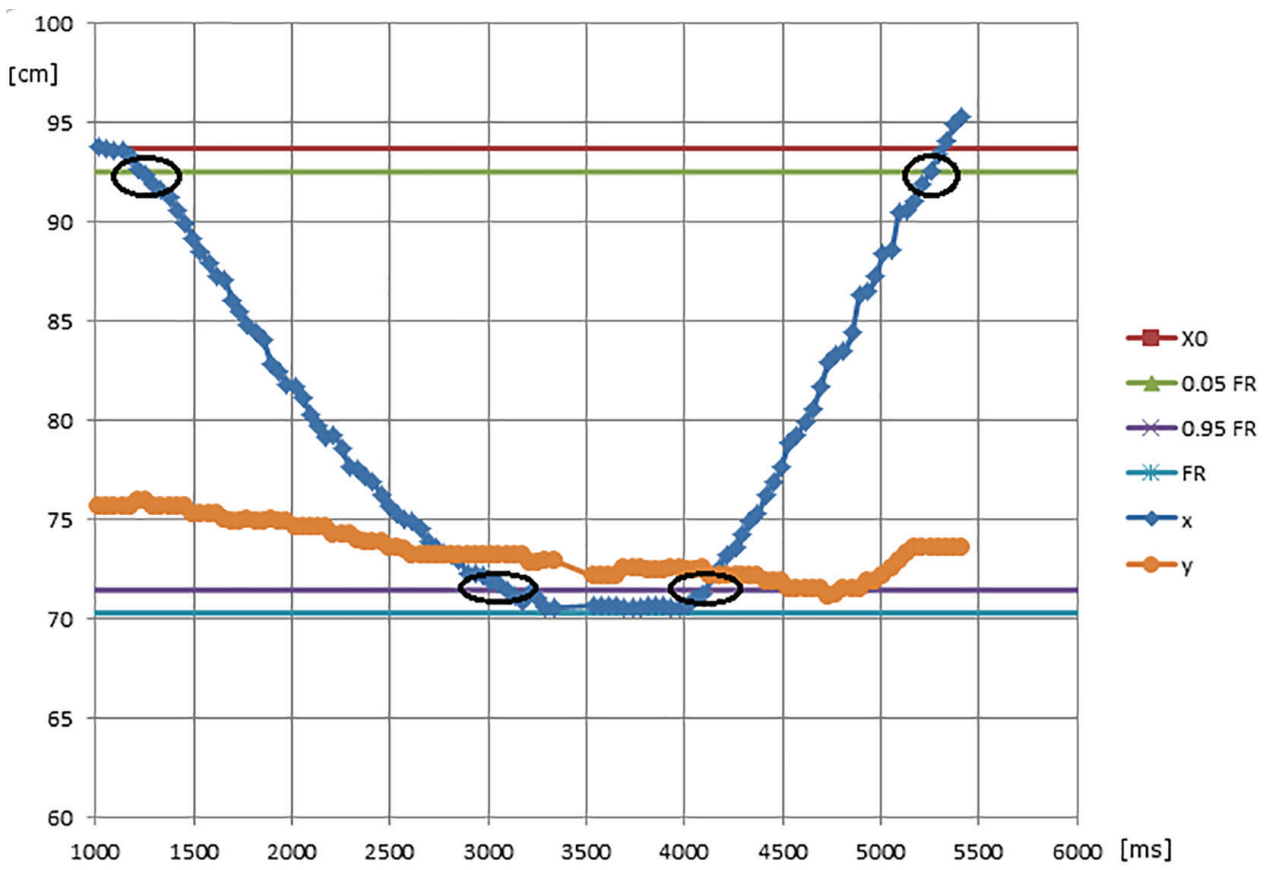

Figure 1. cFRT: wrist displacement trend and characteristic test values (blue line: horizontal trend; orange line: vertical trend; circles at the beginning and at the end: 0.05 FR; circles in the middle: 0.95 FR (forward and backward)).

two sections (balance maximum is 16 and gait maximum is 12). A score of less than 20 indicates a high risk of falls, a score from 20 to 23 indicates risk of falls, from 23 to 27 indicates a very low risk of falls, and 28 is normal. The Tinetti test was administered by a geriatric physician who also evaluated the score of each item.

\section{FRT}

As above mentioned, the FRT is based on analyzing the limits of anterior-posterior stability in an upright position, in the absence of external perturbations. It assesses the maximum forward displacement (in $\mathrm{cm}$ ) that a subject can reach without losing balance.

\section{Description of the FRT}

The subject stands parallel to a wall, close to but not touching it, and with feet pelvic width apart. The arms are positioned at $90^{\circ}$ of flexion, with elbows and hands extended, fingers closed. The subject's dominant arm must be extended forward, always at shoulder level. Then, without moving the feet off the ground, the participant performs hip flexion by moving the trunk forward and reaching as far forward as possible without taking a step and without lowering the arm. The position in which the subject, stretching forward, would be forced to alter his/her base is considered the maximum distance that the subject is able to reach. The wrist should advance, at least $15 \mathrm{~cm}$; smaller distances indicate a significant risk of a fall, while values between 15 and $25 \mathrm{~cm}$ indicate a moderate risk of fall.

$c F R T$

The cFRT was executed in phases. First phase: the FRT was recorded by a Logitech Express Quickcam (resolution $720 \times$ 480) webcam situated $1.5 \mathrm{~m}$ from the subject. Second phase: the recording ensures that the test was executed correctly. Third phase: the recording was processed by apposite LAB view software, which provides the wrist displacement trend by means of a selected target. The system was calibrated by filming a measuring tape $1.5 \mathrm{~m}$ from the camera. This allowed us to calculate the displacement corresponding to each pixel and thus measure the value of the FR. The precision of the FR value, noting the wrist form and the parallax error is estimated to be within $0.5 \mathrm{~cm}$. The room is lit by a single $100 \mathrm{~W}$ bulb. Recording the test and processing the video makes it possible to measure the FR distance and velocity (between $0.05 \mathrm{FR}$ and $0.95 \mathrm{FR}$ ), the "stop time" (between 0.95 FR forward and 0.95 FR back to the upright position, and eventual arm lowering). Figure 1 shows a test recording, highlighting the horizontal and the vertical trends, and the $0.05 \mathrm{FR}$ and the $0.95 \mathrm{FR}$ values. The test was repeated three times and the mean values of the FR, velocity, stop time and the lowering values for each subject were used.

\section{Statistical analysis}

Statistical analysis was performed using SPSS Version 17. Demographic and clinical data are represented by mean and SD or frequencies. P-values were calculated using Student's 
Table 1. Results of the Tests on the 54 Subjects With Neurological Disorders

\begin{tabular}{llllllll} 
& Age (years) & MMSE (/30) & Tinetti $(\mathbf{2 8})$ & FR $(\mathbf{c m})$ & Velocity $(\mathbf{c m} / \mathbf{s})$ & Stop time $(\mathbf{s})$ & Lowering $(\mathbf{c m})$ \\
\hline Mean & 79.6 & 25.5 & 22.7 & 20.7 & 4.9 & 1.1 & 15.5 \\
SD & 6.1 & 3.6 & 6.9 & 7.7 & 2.5 & 0.7 & 9.5 \\
\hline
\end{tabular}

FR: forward reach; SD: standard deviation.

$t$-test, ANOVA, and Pearson's correlation coefficient was used to indicate the strength of a linear relationship between cFRT and MMSE test. Quantitative FR parameters were compared between patients and healthy control subjects using Student's $t$-test after testing for normal distribution.

\section{Results}

Table 1 shows the values of the characteristics measured while testing the elderly subjects with neurological disorders (outpatients of the Israelitic Hospital of Rome). Particular attention was given to the trend FR-Tinetti score, as both tests assess balance stability (Fig. 2). Many subjects scored the maximum (28 points) on the Tinetti test, while there was a significant dispersion among the FR values.

The correlations between FR and velocity, stop time and arm lowering were $0.60,0.05$ and 0.14 , respectively. These correlations come from single values. Correlations between the cFRT parameters and the other test results were FR-MMSE 0.24 , FR-Tinetti score 0.38 and MMSE-stop time 0.04. The correlations between FR and the other FRT outcomes, except for velocity were low, probably because each parameter is an index of regular or irregular behavior. The correlation between the MMSE score and FRT outcomes was also low, probably because cognitive ability may be completely different from physical performance among subjects with neurological disorders. The correlation between FRT and Tinetti score was not high, although both tests are indices of balance ability. Examining the FRT-Tinetti test correlation in more detail, we considered the values of the FR by dividing the subjects into four groups based on MMSE and Tinetti scores: 1) MMSE $<25$, $\mathrm{T}<25,13$ subjects, $\mathrm{FR}=13.1 \pm 4.7 \mathrm{~cm} ; 2) \mathrm{MMSE}>25, \mathrm{~T}<$ $25,9$ subjects, FR $=21.6 \pm 5.5 \mathrm{~cm} ; 3)$ MMSE $<25, \mathrm{~T}>25$, 10 subjects, $\mathrm{FR}=24.5 \pm 10.3 \mathrm{~cm} ; 4) \mathrm{MMSE}>25, \mathrm{~T}>25,22$ subjects, FR $=23.8 \pm 5.9 \mathrm{~cm}$.

Except for group A, the most vulnerable subjects, which was significantly different from the other three $(\mathrm{P}>0.95)$, a considerable dispersion of the test results was observed (Figs. 3 and 4).

All healthy subjects were more than 70 years of age (mean of $75.9 \pm 4$ ). The outcomes were functional reach distance 23.8 $\pm 4.5 \mathrm{~cm}$, velocity time $28.4 \pm 3.5 \mathrm{~cm} / \mathrm{s}$, stop time $0.505 \pm 0.05$ $\mathrm{s}$, and arm lowering $6.2 \pm 1.3 \mathrm{~cm}$. There were significant differences between the subjects with neurological disorders and the healthy subjects: subjects with neurological impairments performed considerably poorer than the healthy participants; the significance of the difference is $95 \%$ both for the FR and for the MMSE. In the same comparison, the FR-MMSE correlation was smaller in the subjects with diseases $(0.24)$ than in the healthy subjects $(0.44)$. Similarly, comparing the subjects with neurological disorders with the healthy subjects from the nursing homes, the significance of the difference was $95 \%$ for the FR and $99 \%$ for the other FRT parameters.

\section{Discussion}

A significant finding of this study is that except for velocity, subjects with neurological disorders showed low correlations between FR and the other characteristics of the FRT, such as stop time and arm lowering. This means that each parameter

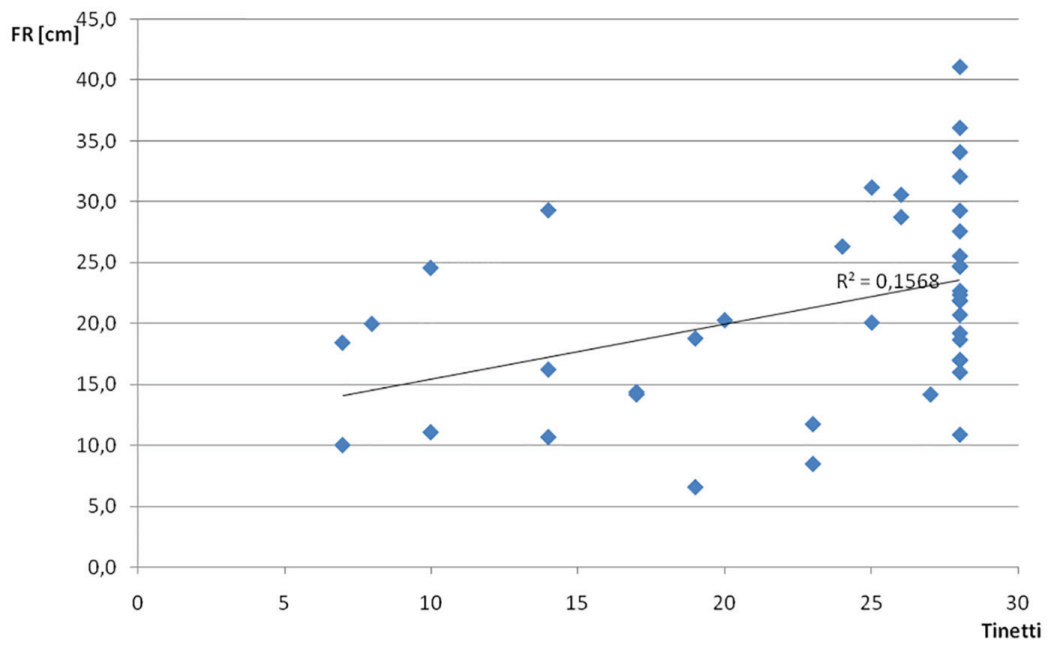

Figure 2. Trend of FR and Tinetti scores. 


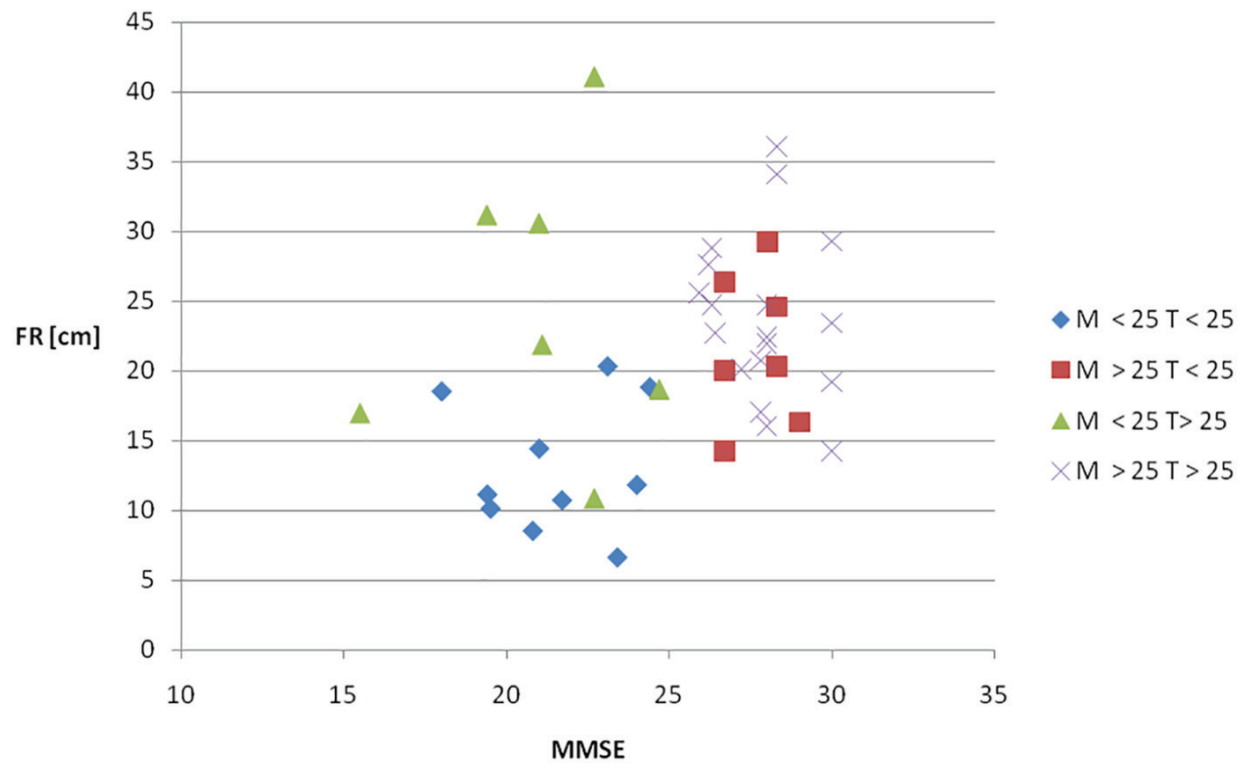

Figure 3. Trends of FR, Tinetti and MMSE scores.

can be considered an index of a particular aptitude: a correct, partially correct, or incorrect behavior. Low correlation was also observed between FR and MMSE and Tinetti scores. As mentioned above, we have been particularly puzzled that while many participants achieved a maximum Tinetti score (28 points), FR values were much more variable. We express some reservations about the validity of the Tinetti test measured by a certain score. The Tinetti test is the most frequently cited balance assessment tool. It has been used to assess mobility dysfunction in the elderly and is an important test for measuring fall risk among various populations [19]. Yet, there are different versions of the Tinetti test with different scoring methods, which make it challenging to assess its validity and reliability.

The FR-MMSE correlation of the subjects with neurological disorders was very low compared to that of the healthy subjects of the Institutes for the Elderly of Rome. It is possible that for the healthy subjects, the physical and the cognitive decline

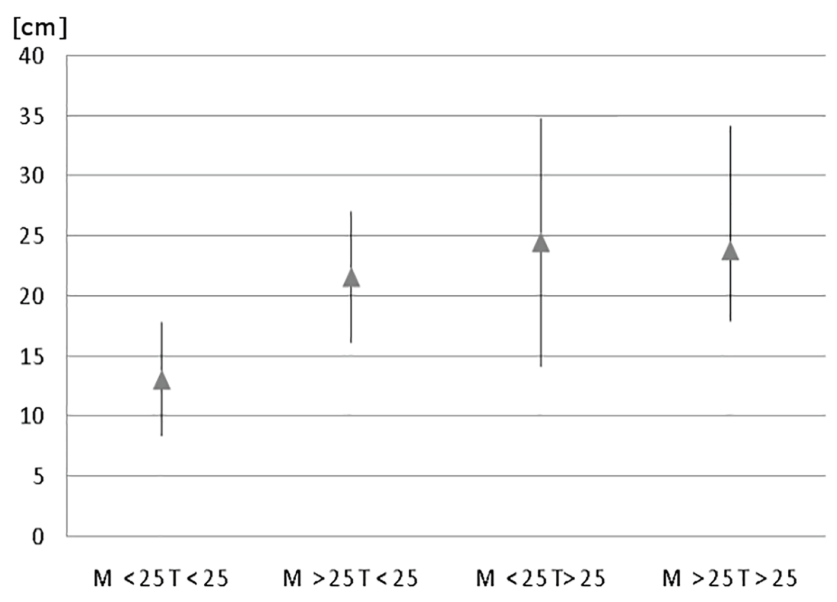

Figure 4. FR mean values (in $\mathrm{cm}$ ) in the four groups. are happening in parallel; whereas, the subjects with diseases decline in a different way, which may indicate that each group requires special attention.

The significant differences found between the performance of the patients with neurological disorders and the healthy control participants require intense discussion. The kinematic values and the experimental usefulness of recording and processing the video of the FRT are important. A slower velocity and a longer stop time are excellent indices of movement difficulties, and to better understand how to perform FRT. Lowering the arm or bending down while reaching forward can serve as a good index of self-confidence [20] and ability to maintain balance while standing. Thus, the lower a person bends down, the poorer the self-confidence when performing a dynamic balance task.

One still must remember that head trauma is quite different from normal aging process, and affects differently physical and mental abilities. Therefore, to apply the findings to general elderly population should be done carefully and with some reservations.

\section{Clinical implications}

We suggest that to assemble a better understanding of a person's ability to control standing balance during FR test, and to enhance prediction of the likelihood of falls among older adults, the therapist should utilize camera motion capture system with synchronized for PC software. By using computerized technology, we can analyze two categories: kinematic effects (motion control) and kinetic effects (joint moments).

A limitation of this study was that we focused only on certain kinematic parameters, such as reach distance, velocity, stop time, and arm lowering. However, cFRT can also yield information regarding reaction time (unexpected command to do FRT), 
time length (from start to maximum reaching forward), and recoil (over backward). We did not allow the subjects to use both arms to perform FRT. Performing FRT with both arms extended forward changes the center of gravity, which can interfere with postural control. Third, the testing protocol only allowed ankle sway synergy (postural adjustments at the ankle joint). We did not allow hip sway synergy (postural adjustments at the hip), and suspensory synergy (flexion at the hip, knee, and ankle) to lower the center of gravity toward the base of support.

In summary, the performance values established in this study indicate that the cFRT is a reliable and valid assessment and a valuable tool for dynamic balance treatment. Here we described a simple procedure of performing the FRT. Instead of manual measurement, we proposed to use a cheap with good resolution web camera to accurately estimate the FRT. Future research should include the two common movement strategies of hip sway synergy and suspensory synergy, used in postural sway that could provide additional, valuable information about dynamic balance control.

\section{Acknowledgments}

This work has been effected thanks to an opposite contribution of the Israelite Hospital of Rome. We also thank the management of the Hospital for making the facilities available during the testing.

\section{Disclosure}

The authors have no conflicts of interest to declare. The study was not funded.

\section{Author Contributions}

All authors (Silvio Scena, Roberto Steindler, Moira Ceci, Stefano Maria Zuccaro, and Eli Carmeli) were equally involved in the study concept and design. Roberto Steindler and Eli Carmeli were also involved in acquisition of subjects and/or data, analysis and interpretation of data, and preparation of manuscript.

\section{References}

1. Okonkwo OC, Griffith HR, Copeland JN, Belue K, Lanza S, Zamrini EY, Harrell LE, et al. Medical decisionmaking capacity in mild cognitive impairment: a 3-year longitudinal study. Neurology. 2008;71(19):1474-1480.

2. Spirduso WW. Physical fitness, aging, and psychomotor speed: a review. J Gerontol. 1980;35(6):850-865.

3. Stelmach GE, Worringham CJ. Sensorimotor deficits related to postural stability. Implications for falling in the elderly. Clin Geriatr Med. 1985;1(3):679-694.

4. Podsiadlo D, Richardson S. The timed "Up \& Go": a test of basic functional mobility for frail elderly persons. J Am Geriatr Soc. 1991;39(2):142-148.

5. Berg K, Wood-Dauphinee S, Williams JI. The Balance Scale: reliability assessment with elderly residents and patients with an acute stroke. Scand J Rehabil Med. 1995;27(1):27-36.

6. Luukinen H, Koski K, Laippala P, Kivela SL. Predictors for recurrent falls among the home-dwelling elderly. Scand J Prim Health Care. 1995;13(4):294-299.

7. Nevitt MC, Cummings SR, Kidd S, Black D. Risk factors for recurrent nonsyncopal falls. A prospective study. JAMA. 1989;261(18):2663-2668.

8. Duncan PW, Weiner DK, Chandler J, Studenski S. Functional reach: a new clinical measure of balance. J Gerontol. 1990;45(6):M192-197.

9. Zur O, Shaki T, Carmeli E. Concurrent Validity and Reliability of a New Balance Scale Used in Older Adults. Adv Exp Med Biol. 2016;910:63-70.

10. Bohannon RW. Measurement of gait speed of older adults is feasible and informative in a home-care setting. J Geriatr Phys Ther. 2009;32(1):22-23.

11. Ramos-Pichardo JD, Cabrero-Garcia J, Gonzalez-Llopis L, Cabanero-Martinez MJ, Munoz-Mendoza CL, Sanjuan-Quiles A, Richart-Martinez M, et al. What do older people understand by mobility-related difficulties? Arch Gerontol Geriatr. 2014;59(1):122-130.

12. Lin MR, Wolf SL, Hwang HF, Gong SY, Chen CY. A randomized, controlled trial of fall prevention programs and quality of life in older fallers. J Am Geriatr Soc. 2007;55(4):499-506.

13. Katz-Leurer M, Fisher I, Neeb M, Schwartz I, Carmeli E. Reliability and validity of the modified functional reach test at the sub-acute stage post-stroke. Disabil Rehabil. 2009;31(3):243-248.

14. Costarella M, Monteleone L, Steindler R, Zuccaro SM. Decline of physical and cognitive conditions in the elderly measured through the functional reach test and the mini-mental state examination. Arch Gerontol Geriatr. 2010;50(3):332-337.

15. Zuccaro SM, Steindler R, Scena S, Costarella M. Changes of psychical and physical conditions in the elderly after a four-year follow-up. Arch Gerontol Geriatr. 2012;54(1):72-77.

16. Carmeli E, Katz LM, Scena S, Kodesh E, Steindler R. Functional reach test performance in distance and velocity - A pilot study. Eur J Physiother. 2014;16(2):168-172.

17. Folstein MF, Folstein SE, McHugh PR. "Mini-mental state". A practical method for grading the cognitive state of patients for the clinician. J Psychiatr Res. 1975;12(3):189-198.

18. Tinetti ME, Williams TF, Mayewski R. Fall risk index for elderly patients based on number of chronic disabilities. Am J Med. 1986;80(3):429-434.

19. Kopke S, Meyer G. The Tinetti test: Babylon in geriatric assessment. Z Gerontol Geriatr. 2006;39(4):288-291.

20. Adkin AL, Frank JS, Carpenter MG, Peysar GW. Fear of falling modifies anticipatory postural control. Exp Brain Res. 2002;143(2):160-170. 